Musées, Patrimoine et Culture scientifiques et techniques

$171 \mid 2017$

mai-juin 2017

\title{
30 ans après, le "journalisme d'exposition" vu par Science Actualités
}

Alain Labouze

\section{OpenEdition}

\section{Journals}

Édition électronique

URL : http://journals.openedition.org/ocim/1790

DOI : $10.4000 /$ ocim. 1790

ISSN : 2108-646X

Éditeur

OCIM

Édition imprimée

Date de publication : 1 mai 2017

Pagination : $34-37$

ISSN : 0994-1908

Référence électronique

Alain Labouze, «30 ans après, le "journalisme d'exposition" vu par Science Actualités », La Lettre de I'OCIM [En ligne], 171 | 2017, mis en ligne le 01 mai 2018, consulté le 22 avril 2019. URL : http:// journals.openedition.org/ocim/1790 ; DOI : 10.4000/ocim.1790

Ce document a été généré automatiquement le 22 avril 2019

Tous droits réservés 


\title{
30 ans après, le "journalisme d'exposition" vu par Science Actualités
}

\author{
Alain Labouze
}

L'article disponible en ligne sur www.ocim.fr (rubrique La Lettre de l'OCIM) montrait comment les centres de science peuvent porter un autre regard sur l'actualité scientifique, différent de celui des autres organes de presse.

Vers un " journalisme d'exposition "

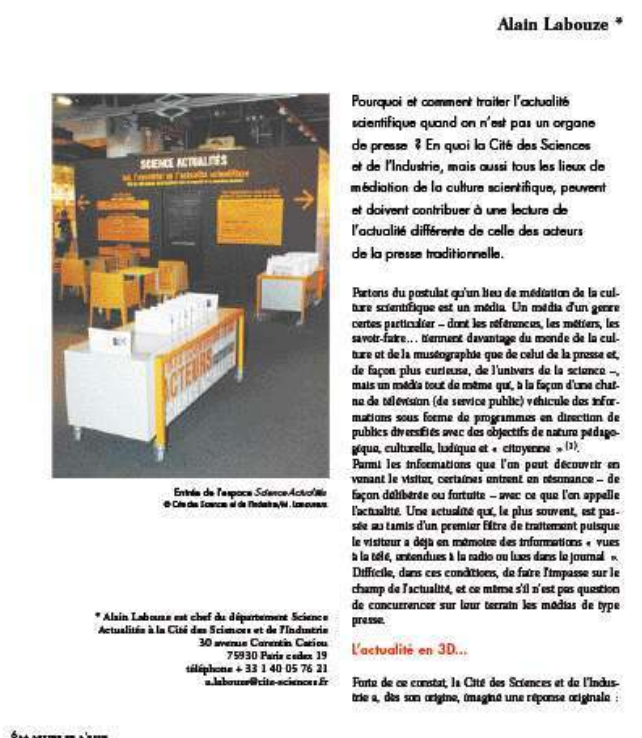


1 "Pourquoi et comment traiter l'actualité scientifique quand on n'est pas un organe de presse ?" : telle était la question posée en 2004 dans un numéro spécial de La Lettre de l'OCIM consacré à la place de l'actualité dans les centres de science. Aujourd'hui, la Cité des Sciences et de l'Industrie contribue toujours à une lecture originale de l'actualité par rapport à celle des acteurs de la presse traditionnelle.

2 Comment proposer un décryptage de l'actualité scientifique sur un mode journalistique différent de celui des acteurs de la presse traditionnelle ? Dès sa création, la Cité décidait de dédier un espace muséographique à l'actualité scientifique, une "exposition d'actualité" en forme d'oxymore au sens qu'elle serait permanente mais à renouvellement constant. Depuis 30 ans - de 1986 jusqu'à aujourd'hui -, la Cité a donc fait vivre un espace d'exposition (nommé Science Actualités pour rendre évidente l'analogie avec la presse) et ainsi a pu proposer à ses visiteurs une offre d'actualité regroupée.

Durant toute cette période, la Cité a expérimenté différentes formules. Des changements ont été opérés aussi bien au plan éditorial que muséographique ou encore géographique, en déplaçant la "salle d'actualités" dans divers lieux du musée : hall de la Cité, bibliothèque, au cœur des expositions d'Explora (site actuel).

En 2017, l'objectif du média exposition Science Actualités reste naturellement d'informer les publics de la Cité sur l'essentiel des sujets d'actualité scientifique et ainsi de faciliter la compréhension des enjeux liés à l'évolution des sciences et des techniques. Avec, par rapport à ses débuts, un accent particulier mis sur les flux d'informations et la place de la vidéo, l'ouverture aux sujets européens et mondiaux, la participation accrue des scientifiques et l'interactivité avec les visiteurs, laquelle s'est matérialisée notamment par un dispositif audiovisuel de recueil d'opinions.

5 Ajoutons qu'en termes de process, et c'est un point essentiel pour répondre à la question fondamentale du "qui parle ?", la production des contenus est toujours confiée à une rédaction composée de journalistes scientifiques. Non pas que les journalistes détiennent le monopole du traitement de l'actualité, mais ils obéissent à des règles de fonctionnement et de déontologie qui permettent à ce média atypique de s'appuyer, à travers l'existence d'un comité éditorial, sur un partenariat fécond avec l'AJSPI (association des journalistes scientifiques de la presse d'information). Il s'agit de guider les choix éditoriaux et veiller à la qualité des contenus produits et présentés au public : recoupement des informations, diversité des sources, travail d'enquête, regard critique, circuit de validation... autant de fondamentaux du métier de journaliste, garants a priori d'une information rigoureuse, fiable, vérifiée et indépendante.

\section{Une offre d'actualité dans tous les centres de science} ?

6 Au-delà de la Cité des Sciences et de l'Industrie et des quelques autres centres de science pionniers en la matière, peut-on imaginer que les professionnels de la médiation scientifique en régions s'emparent de l'expérience réussie de Science Actualités pour proposer une offre d'actualité scientifique et technologique de type journalistique?

7 En partenariat avec la presse quotidienne régionale $(\mathrm{PQR})$ et avec les antennes en régions de Radio France, ne pourrait-on pas construire un réseau de correspondants "Actualités" permettant de mutualiser les informations en provenance des différents territoires? 
Peut-être serait-ce là le moyen d'élargir la réflexion sur les pratiques journalistiques et les moyens de mettre en œuvre une pédagogie critique de l'information?

Grâce aux bourses de coproduction Estim-Numérique, nous avons pu ces dernières années mutualiser des moyens dans le domaine de la production audiovisuelle et multimédia scientifique. Avec à l'arrivée plusieurs centaines de films courts, reportages, émissions TV, documentaires, conférences filmées, webdocs, serious games... mis à la disposition de tous les acteurs du réseau de CST, et pouvant répondre à des besoins en muséologie, en médiation humaine ou à des activités en ligne.

9 La récente décision par le président d'Universcience d'une mise à disposition aux centres de culture scientifique, technique et industrielle (CCSTI), par voie dématérialisée, de toutes les productions de l'espace Science Actualités pourrait constituer une nouvelle étape ambitieuse de mutualisation des contenus dans le domaine de l'actualité scientifique.

\section{L'actualité scientifique : une définition}

10 Mais avant d'imaginer un futur collectif et dématérialisé, commençons par (re)préciser de quoi l'actualité est le nom... Par provocation, certains spécialistes en communication avancent que l'actualité n'existe pas, ou du moins que pour accéder au statut d'actualité et encore plus d'événement - une information doit d'abord être relayée par les médias.

11 De notre point de vue, rendre compte de l'actualité scientifique revient à informer sur :

12 - ce qui est nouveau (découvertes, avancées, annonces...). Le suivi de l'actualité des recherches menées dans les laboratoires, qui donnent lieu à des publications dans des revues scientifiques, est essentiel pour rendre compte des progrès observés, et ce même si les "petits pas de la science" sont, on le sait, rarement spectaculaires;

13 - ce dont on parle (dans les médias traditionnels, et qui mérite décryptage et analyse critique). Les publics sont imprégnés de ce que les médias disent d'un certain nombre de sujets d'actualité. Il est impossible de ne pas tenir compte du "vu à la télé", "entendu à la radio", "lu dans la presse" ou "découvert sur Internet ou les réseaux sociaux";

14 - ce qui pose questions (quand les avancées de la science et de la technologie imposent débats et prises de position; quand la science peut apporter des éclairages particuliers sur certains événements ou certaines questions a priori hors champ scientifique). Sont concernés au premier chef, d'une part, les grands feuilletons d'actualité sur des sujets à controverses et, d'autre part, les "actualités du monde" dont le décryptage peut être facilité par le recours à une expertise scientifique : événements prévisibles (grandes compétitions sportives, élections...) ou imprévisibles (catastrophes naturelles, guerres...) ;

15 - ce qui permet de témoigner de la réalité du terrain (dans les lieux où se fait la science au quotidien, où se fabrique l'information scientifique...). L'incarnation par les femmes et les hommes qui font avancer la science rend cette dimension de l'actualité scientifique particulièrement humaine et accessible au plus grand nombre. Par ailleurs, le décryptage de la façon dont est produite l'information, notamment dans les médias, permet d'aiguiser le regard critique porté sur l'actualité.

16 Au plan plus académique, l'actualité scientifique est un terme générique qui recouvre en fait de vastes champs thématiques, en particulier la santé, l'environnement, la 
technologie, le numérique, et bien sûr les sciences au sens le plus large du terme qui englobe les sciences humaines.

Aujourd'hui l'espace Science Actualités est un espace d'exposition muséographié : ici le "Mur de news".

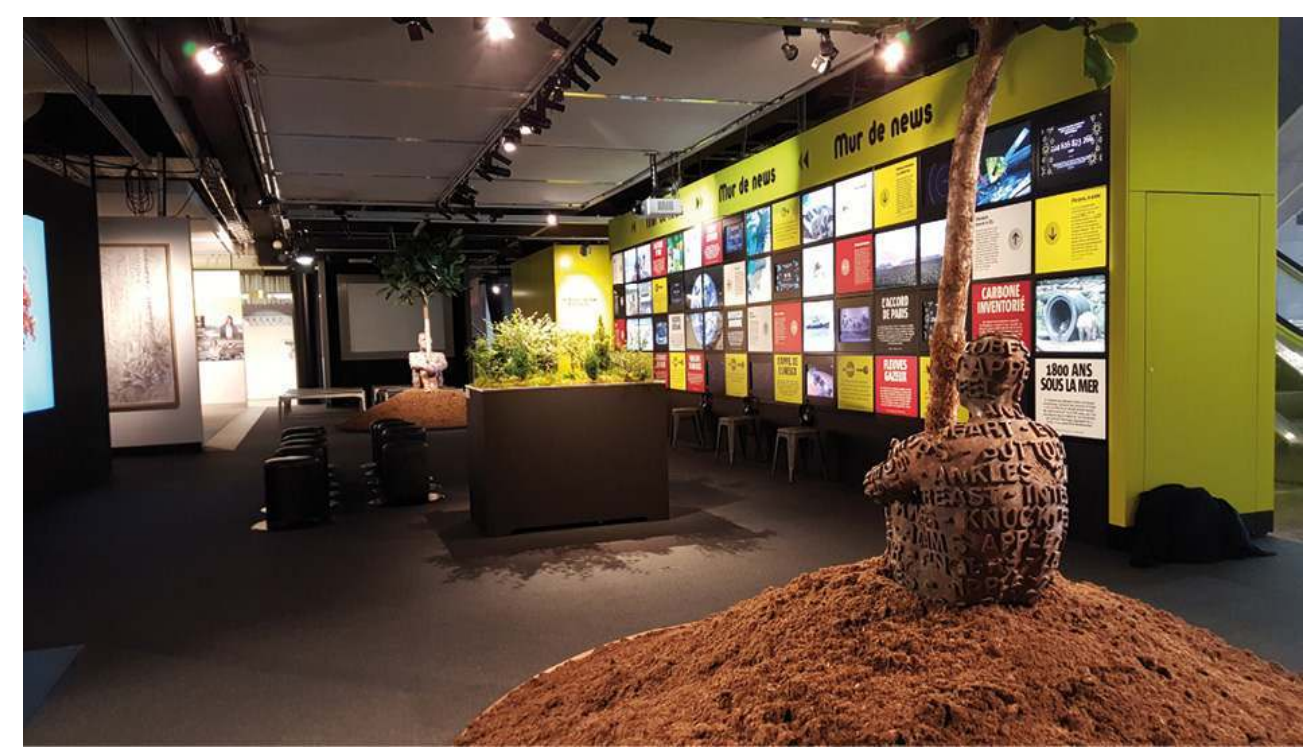

(c) Universvience/Jérôme Kazazian

\section{Le projet muséographique actuel à la Cité}

17 Afin que chacun puisse évaluer si une transposition des contenus exportés de Science Actualités peut présenter un intérêt dans le contexte local, une description s'impose.

Aujourd'hui l'espace Science Actualités est un espace d'exposition conçu d'un point de vue muséographique avec des gabarits contraints, le format proposé se déclinant en quatre sous-ensembles qui correspondent à la fois à des postures particulières de visiteurs, à des axes éditoriaux forts (rubriques) et à des temporalités différentes.

\section{Questions d'actualité}

19 Quatre grandes enquêtes sont traitées sous la forme de panneaux graphiques (français, anglais), quiz multimédia ainsi que vidéo et interview audio intégrées aux cimaises. Les Questions d'actualité sont renouvelées tous les quatre mois, soit 12 sujets par an. Parmi les dernières Questions d'actualité, on retrouve : "Darknet, la face cachée du web", "ISS, quel bilan scientifique ?", "Résistance antibiotique, un fléau négligé", "Pomme de terre, la reine des Andes", "L’animal est-il une personne ?", "Trous noirs, une étrangeté cosmique", "Microbiote, une révolution médicale", "Moustiques, la guerre est déclarée", "Préhistoire : les migrants du passé", "Santé : la vaccination en débat", "Chaco : une forêt en sursis", "CRISPR-Cas9 : la révolution génétique"... 


\section{Mur de news}

Cette composition graphique géante de 64 panneaux intègre brèves de l'actualité scientifique, images de science, compteurs (dimension "observatoire") permettant un suivi en direct d'un certain nombre d'indicateurs de la planète, vidéos du "ciel de la semaine" et du "Journal de la semaine" (infos principalement tirées des publications scientifiques). Ce mur de news est construit à la manière de casses d'imprimerie.

\section{Chercheurs à la une}

Une installation audiovisuelle propose reportages et interviews, qui rendent compte de la science en train de se faire dans les laboratoires ou lors de missions scientifiques, ainsi que des enjeux sociétaux liés aux avancées scientifiques, avec des réactions et commentaires de scientifiques et experts aux actualités du moment.

\section{Points de vue d'artistes}

Une exposition d'œuvres d'art en lien avec l'une des Questions d'actualité offre une carte blanche à des artistes qui s'emparent des contenus de l'actualité scientifique pour livrer leurs visions de la science, de la technologie et d'un monde en mutations.

C'est la place accordée à l'imaginaire, au sensible, parfois aux questionnements dérangeants ou au prospectif. La place aussi des objets dans un "magazine-exposition" qui se visite.

Tel un magazine scientifique en 3 dimensions qui présente des œuvres en miroir pour questionner l'actualité scientifique et technologique traitée par la rédaction de Science Actualités...

Les œuvres n'étant pas prévues pour circuler, à chaque centre de science de décider s'il souhaite aussi associer une exposition artistique en complément des contenus proposés.

Des numéros spéciaux peuvent en outre être intégrés dans la scénographie existante de l'espace Science Actualités. Cela a été le cas avec Climat, l'expo à $360^{\circ}$ (à l'occasion de la COP21), Fukushima, l'hommage rendu aux dessinateurs de presse ou les résultats de la mission scientifique Makaï à Madagascar.

\section{L'offre numérique d'actualité scientifique}

Reste l'apport du numérique qu'il convient de ne pas sous-estimer... Outre l'accès aux contenus de Science Actualités via son site internet, la webTV universcience.tv propose depuis 2010 une programmation originale $100 \%$ vidéo, renouvelée chaque semaine, accessible à tous les publics et riche d'un fonds de plus de 3800 films (en accès gratuit).

Avec la création du "pôle information" de cette webTV, à laquelle contribue une trentaine de partenaires institutionnels, organismes de recherche et centres de science, Science Actualités a augmenté son volume de production audiovisuelle en lien avec l'actualité scientifique : plus de reportages pour rendre mieux compte des avancées scientifiques et du travail des chercheurs sur le terrain ; plus d'interviews et des débats pour aborder les questions "sciences et société" ; un "Journal des sciences" pour présenter les derniers 
travaux de recherche en images ; et les clés de l'observation hebdomadaire de la voûte céleste ("Ciel !"). Le "Journal des sciences" et le "Ciel !" hebdomadaires sont diffusés dans le Mur de news et les reportages et interviews dans la zone Chercheurs à la une de l'espace d'exposition Science Actualités. Quant aux films coproduits dans le cadre des bourses Estim-Numérique, ils permettent de nourrir la programmation d'universcience.tv.

29 À l'évidence, l'avenir de la présentation dans nos centres de science de contenus liés à l'actualité scientifique est de bien relier le média exposition aux offres numériques satellites. Une optimisation des contenus qui va de pair avec la réflexion en cours sur les nouvelles formes d'écriture.

INDEX

Mots-clés : journalisme, exposition

\section{AUTEUR}

\section{ALAIN LABOUZE}

Chef du département Actualités et Audiovisuel, rédacteur en chef de Science Actualités, responsable d'universcience.tv, Universcience

alain.labouze@universcience.fr 